\title{
Effect of Pulse Electric Current Stimulation on the Microstructure, Mechanical Properties and Thermal Fatigue Behavior of Cast-hot-working Die Steel
}

\author{
Huaqiang LIN, ${ }^{1)}$ Yuguang ZHAO, ${ }^{11}$ Yang $\mathrm{ZHAO}^{2)}$ Liguang HAN, ${ }^{1)}$ Jun MA ${ }^{11}$ and Qichuan JIANG ${ }^{11}$ \\ 1) Key Laboratory of Automobile Materials of Ministry of Education and Department of Materials Science \& Engineering, Jilin \\ University, No. 142 Renmin Street, Changchun 130025, P. R. China. 2) School of Communication Engineering, Jilin \\ University, No. 142 Renmin Street, Changchun 130025, P. R. China. E-mail: zhaoyg@mail.jlu.edu.cn
}

(Received on November 1, 2007; accepted on December 3, 2007)

\begin{abstract}
The microstructure variations in the heat affected zone (HAZ) of cast hot working die (CHWD) steels initiated by pulse electric current stimulation were investigated by the transmission electron microscopy (TEM) observation. Under the function of pulse electric current stimulation, some carbide particles segregating on the boundaries of neighboring lathing martensite in the original steel, were diffused as solid solution phases to the inner of martensite matrix. Compared with carbide agglomeration precipitating with irregular shape in the fatigued steel after 500 thermal fatigue cycles, the globularity of carbide was enhanced by degree and the interface between carbide and matrix was much improved in the corresponding electrostimulated steel. The X-ray diffraction analysis showed that the dislocation density was obviously increased in the HAZ when the application of pulse electric current through the original steel, especially for the stimulated specimen after thermal fatigue for 500 cycles. The reason was regarded as that the dislocation motion and multiplication were enhanced by the electro-dislocation interaction, Joule heating effect and thermal compressive stress under the function of pulse electric current stimulation. By the coexisting action of strengthening mechanisms by refining grain, increasing dislocation density and dispersively distributing nanosized carbide particles, the ultra tensile strength (UTS) of the HAZ was improved from $1738 \mathrm{MPa}$ in original state to $2420 \mathrm{MPa}$ by the stimulation of pulse electric current. Especially for the stimulated steel after thermal fatigue for 500 cycles, the UTS even reached $2687 \mathrm{MPa}$. Pulse electric current stimulation played a strengthening role on CHWD steel material and inevitably improved the thermal fatigue behavior and prolonged the service lifetime of die material.
\end{abstract}

KEY WORDS: pulse electric current; microstructure; UTS; thermal fatigue; CHWD steel.

\section{Introduction}

The hot working dies, called as "black gold" for their complicated machining process and high cost, play an important role in liquid metal forming operations. Because of service in extreme conditions, the die material experiences a cyclic process of alternate heating and cooling. The alternate expansion and contraction across the sections are inhomogeneous and produce a system of concentrated stress. Thermal fatigue cracking would initiate at the location with stress concentration and propagate along the grain boundary. ${ }^{1)}$ The network of tiny cracks forming on the exposed surfaces to thermal atmosphere not only damages the surface quality of die but also degrades the surface of the casting. ${ }^{2)}$ More than $80 \%$ of the failure of die was caused by the thermal fatigue. Therefore, how to improve the resistance against thermal fatigue of the die material and delay the initiation and propagation of thermal fatigue cracking is concerned by material researchers up to now.

It is well known that the external pulse electric current stimulation can be the universal means for change of physi- cal-mechanical properties of materials. Pulse electric current, as a novel technique which spreads up increasingly in material processing during recent years, is widely applied to improve the microstructures and properties of materials. The application of pulse electric current in the field of material is manifested in the following aspects: the control of solidification structure, ${ }^{3-6)}$ solid state phase transformation and recrystallization of nonferrous metals, ${ }^{7-9)}$ preparation of nanocrystalline, ${ }^{10-13)}$ eliminating the embrittlement of amorphous alloys and modifying plastic deformation, ${ }^{14-16)}$ improving the fatigue of metals and healing the damage of steels. ${ }^{17-21)}$ However, the acting mechanism of pulse electric current has not been completely explicit in respect of improving the microstructure and properties of materials, especially for improving the resistance against thermal fatigue.

The improvement of pulse electric stimulation on the thermal fatigue resistance of CHWD steel has been experimentally verified from the viewpoint of grain refinement in our previous works. ${ }^{22,23)}$ The purpose of this study is to further investigate the effect of pulse electric stimulation on 
the microstructure evolution and mechanical properties of CHWD steel. The possible mechanism is illuminated for further understanding the nature of improving thermal fatigue properties under the action of pulse electric current stimulation.

\section{Experimental Procedures}

\subsection{Material and Sample Preparation}

The experimental material was a high-Cr CHWD steel developed by Jilin University. The typical chemical compositions were $0.25-0.45 \% \mathrm{C}, 8-10 \% \mathrm{Cr}, 0.5-1.5 \% \mathrm{Mo}$, $0.3-1.0 \% \mathrm{Ni}, 0.6-1.8 \% \mathrm{Si}, 0.4-0.8 \% \mathrm{Mn}$ and Fe balance (all in $\mathrm{wt}^{\mathrm{O}} \%$ ). The preheating stages including two heat preservation treatments at $1080^{\circ} \mathrm{C}$ and $880^{\circ} \mathrm{C}$ for 1 and $3 \mathrm{~h}$ with subsequent oil and furnace cooling, respectively, were carried out on the CHWD steel. The oil quenching was performed with ambient temperature after austenization at $1080^{\circ} \mathrm{C}$ for $1 \mathrm{~h}$. The tempering treatments were composed of double isothermal treatments for $3 \mathrm{~h}$ at $600^{\circ} \mathrm{C}$ and $580^{\circ} \mathrm{C}$ with sequent air cooling, respectively.

The thermal fatigue samples with dimensions of $40 \times$ $12 \times 3 \mathrm{~mm}^{3}$ were machined from the CHWD steel by electric sparkling machine. An additional notch with the gage width of $0.18 \mathrm{~mm}$ and length of $3 \mathrm{~mm}$ was machined at the central section in the longitudinal direction on every specimen for the investigation of thermal fatigue crack. To eliminate stress concentration caused by electric sparkling machining, the surface of the specimen was grinded with $\mathrm{SiC}$ paper of different fineness and subsequently polished on silk fabric with $0.5 \mu \mathrm{m}$ diamond paste.

\subsection{Pulse Electric Current Treatment}

The pulse electric current with the frequency of $50 \mathrm{~Hz}$ was generated from self-made energy-stored equipment under ambient condition. ${ }^{22,23)}$ The specimen was directly connected in the discharging circuit. The programmable controller on the industrial computer commanded the whole discharging process by triggering the silicon controlled rectifier. The average density of electric current passing through specimen transect ahead of notch was approximately $90 \mathrm{MA} / \mathrm{m}^{2}$.

\subsection{Thermal Fatigue Test}

The thermal fatigue test was carried out on a self-controlled thermal fatigue testing machine. ${ }^{22,23)}$ The temperature of the furnace on the thermal fatigue testing machine was almost constant-controlled at about $700^{\circ} \mathrm{C}$. The surface temperature of thermal fatigue sample was associated with the heating time in the furnace. Once the determined heating time reached $60 \mathrm{~s}$ and the surface temperature of specimen approached the tempering temperature, the cantilever instantly moved down and laid the specimens in a flume. The specimens were water-cooled for $4 \mathrm{~s}$ in a flume with flowing water where the temperature was about $20^{\circ} \mathrm{C}$ and again uplifted into the furnace by the cantilever for another heating. The above-mentioned process was controlled automatically and carried out repeatedly. The thermal fatigue cycle number was recorded by an arithmometer on thermal fatigue testing machine.

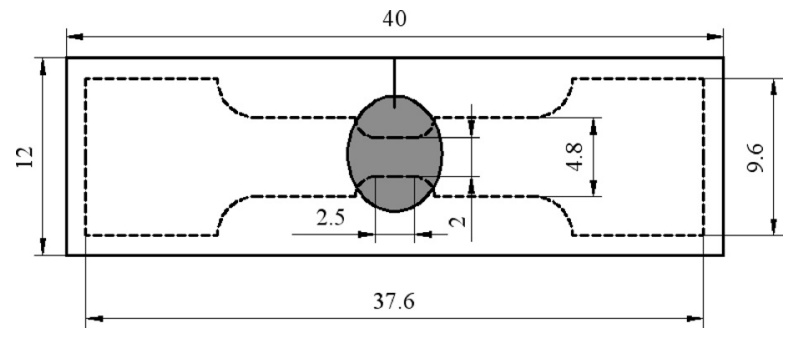

Fig. 1. Schematic illustration for the selection of tensile specimen on thermal fatigue specimen.

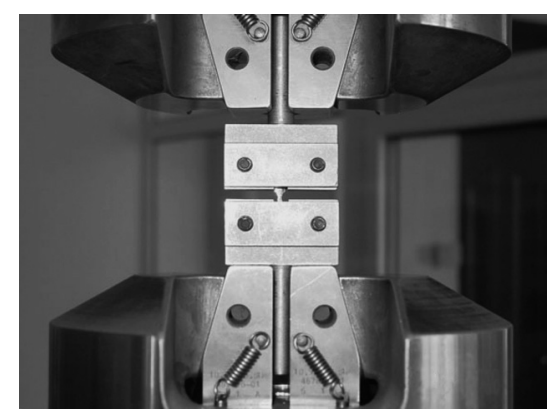

Fig. 2. The installation of tensile specimen on the tensile bar of MTS810.

\subsection{Observation of TEM}

The transmission electron microscopy (TEM) observation of thin foil was performed on a JEM-2000FX instrument with accelerating voltage of $200 \mathrm{kV}$. The round and thin metal films with the thickness of $0.3 \mathrm{~mm}$ were machined by electric sparking machine from the position corresponding to heat affected zone of electropulsed specimens. Different fineness of $\mathrm{SiC}$ papers were used to grind the thin metal films. The thin foils were prepared by electrolytic polishing in an alcohol solution containing $10 \%$ perchloric acid at the cooling atmosphere of liquid nitrogen.

\subsection{Test of Mechanical Properties}

To compare the variation of tensile properties, the tensile samples were machined from the position corresponding to heat affected zone of electropulsed specimens, as illustrated in Fig. 1. The tensile experiment was performed on the material test systems (MTS810). The installation of tensile specimen on the tensile bar was shown in Fig. 2. The displacement velocity of tensile bar on the MTS810 was $0.2 \mathrm{~mm} / \mathrm{min}$. In every group, three samples were selected and tested, and the attained data were calculated to obtain the average value. The tensile curves were analyzed to assess the effect of pulse electric current on the mechanical properties of material.

\section{Results and Discussion}

\subsection{Microstructure Variation in HAZs}

Figure 3 shows the TEM observation results on the thin foil of CHWD steels with different treatments: (a) original and unelectrostimulated $(\mathrm{O}),(\mathrm{b})$ electrositimulation on the original steel (OE), (c) thermal fatigue for 500 cycles (F) and (d) electrostimulation on the fatigued steel for 500 cycles (FE). The CHWD steel in the original state was a polycrystalline aggregate and consisted of martensite and 

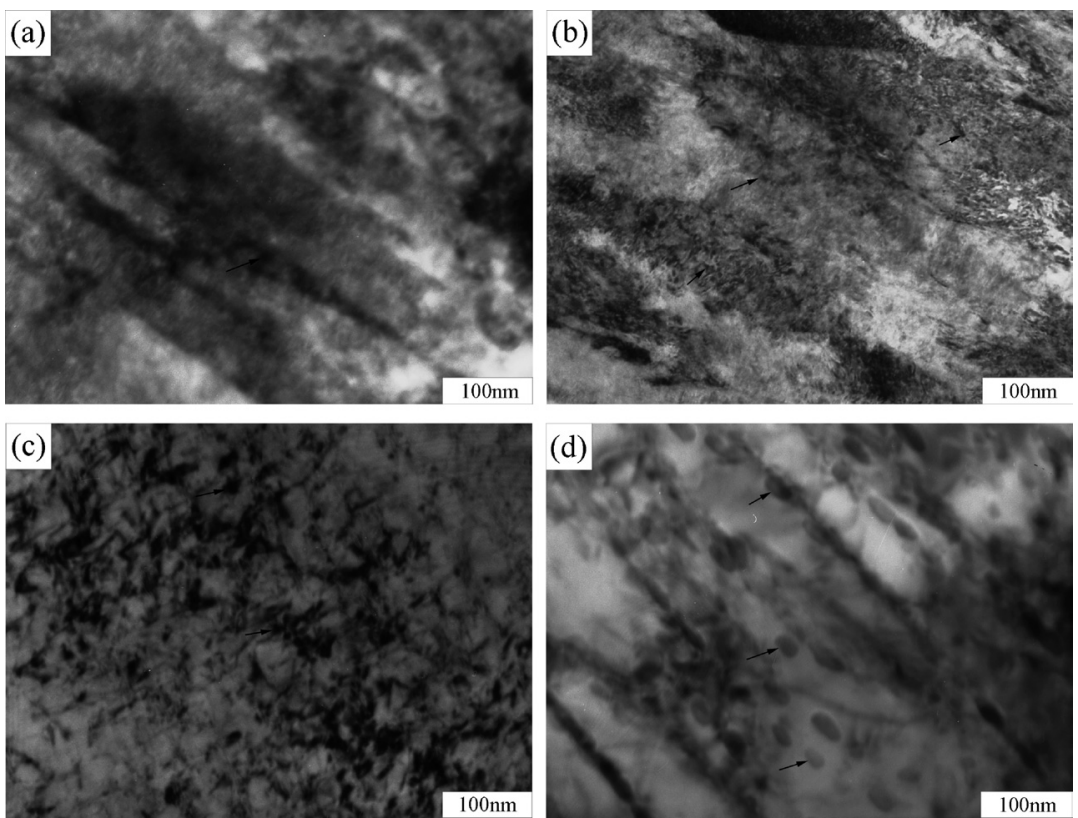

Fig. 3. Transmission electron micrographs of CHWD steels with different treatments: (a) O, (b) OE, (c) F and (d) FE.

carbide particles. The TEM observation shows that the original microstructure of quenched and tempered CHWD steel is tempered lathing martensite bunchily distributing with certain orientation, as illustrated in Fig. 3(a). Some carbide particles segregated and distributed on martensite matrix, especially on the boundaries of neighboring lathing martensites. Pulse electric current stimulation of the CHWD steel initiated the formation of circle or elliptical HAZ (as gray region schematically shown in Fig. 1) and obvious microstructural changes. Due to the existence of the notch on the thermal fatigue specimens, the electric current surpassed and concentrated at the position ahead of the specimen notch, which would produce the concentrated distribution of electric current with high density and result in consequent heat concentration due to Joule heat effect. When the pulse electric current was passing through the notched thermal fatigue specimen, most of electric energy was released as heating in the bulk of the material. During the discharge process of pulse electric current, the region ahead of the notch would instantaneously experience the austenization process with high nucleation rate. After the discharge process of pulse electric current, the rapid cooling of the above-mentioned region would occur because the heat exchange with the matrix at low temperature and restrained the growth of nucleated grains due to the absence of dynamic conditions. The rapid heating and cooling processes consequentially resulted in the formation and grain refinement of HAZ. ${ }^{22,23)}$ The HAZ was represented as a "white-bright layer" ahead of specimen notch, whose appearance was repeatedly noted previously in cases where the concentrated supply of external energy was input, e.g., upon friction or laser or plasma treatment. ${ }^{20)}$ The TEM observation on the HAZ, as depicted in Fig. 3(b), shows that part of carbide particles were redistributed from the boundaries of neighboring lathing martensite to the inner of martensite. The reason was regarded as that the passage of pulse electric current through the specimen was followed by Joule heat release and the Joule heat served as the
Table 1. Result about dislocation density attained by X-ray diffraction test on different specimen HAZs.

\begin{tabular}{ccccc}
\hline Specimen No. & O & OE & F & FE \\
\hline Temperature, ${ }^{\circ} \mathrm{C}$ & - & 879 & - & 921 \\
Dislocation Density, $\mathrm{cm}^{-1}$ & $0.7 \times 10^{12}$ & $3.0 \times 10^{12}$ & $0.9 \times 10^{12}$ & $3.6 \times 10^{12}$ \\
\hline
\end{tabular}

activation energy for the initial carbide particles dissolving in the martensite matrix. During the succedent cooling process, the dissolving carbides dispersively re-precipitated in the martensite matrix. In addition, the electric current was much more sensitive to carbide than martensite, so the electron wind force and thermal compressive stress would propel carbide particles diffusing into martensite matrix. The excessive segregation and precipitation of newly formed carbide particle in the martensite inevitably resulted in the crystal distortion and enhanced the resistance of dislocation movement during the plastic deformation. When the pulse electric current was passing through the bulk of metal material, some accompanying effects, such as electron wind force, thermal release and thermal stress, were induced by pulse electric current stimulation. If the induced thermal stress could not exceed the yield strength of material, the induced energies would promote the mobility of existing dislocations. Once the moving dislocations passed the barriers, such as carbide particles, the dislocation loops were produced in the stimulated specimen. The dislocation multiplication induced by pulse electric current would result in the increase of dislocation density. The X-ray diffraction analysis was performed on the HAZ and the result was enlisted in Table 1. It was showed that the dislocation density increased from $0.7 \times 10^{12} \mathrm{~cm}^{-1}$ in the original state to $3.0 \times 10^{12} \mathrm{~cm}^{-1}$ after by pulse electric current stimulation. Therefore, the strengthening mechanism by increasing dislocation density was inevitably enhanced in the stimulated steel by pulse electric current. During the thermal fatigue process, the plastic deformation was represented by the slip along a certain crystal orientation and the slip band served 
as the channel for dislocation motion due to high activation energy at unstable state. The dislocation movement was obstructed by carbide particles and the dislocation packed up around the carbide. The TEM observation showed that the carbide particles assembled and grew into coarse ones distributing on the boundaries of original neighboring martensites after the thermal fatigue for 500 cycles, as depicted in Fig. 3(c). The driving force for carbide coarsening was regarded as the repeated plastic deformation during the thermal fatigue process. The X-ray diffraction analysis showed that the dislocation density increased to $0.9 \times$ $10^{12} \mathrm{~cm}^{-1}$ after the thermal fatigue for 500 cycles. The coherent interface between coarsening carbide particle and martensite matrix was very irregular in shape. Because the stress concentration was prone to form around carbide particle with the pointed shape, the thermal fatigue crack was liable to generate at the interface between the carbide and matrix. The irregularity of carbide had a negative influence on the thermal fatigue behavior, which inevitably reduced the service lifetime of CHWD steel. Compared with the fatigued specimen after 500 thermal cycles, the corresponding electrostimulated specimen experienced a remarkable microstructure changes in the HAZ. A lot of extraordinary processes, such as the dissolution of solid solution, transfer of carbon atoms from dislocation and other defects, and the formation of carbide-phase particles with globular morphology, were initiated in the material by pulse electric current stimulation in the intermediate stage of thermal fatigue cycle. On one hand, the coarsening carbide particles in the fatigued specimen were broken down under thermal compressive stress and some of them dissolved into martensite matrix under the application of pulse electric current stimulation. The newly formed carbide particles had a pinning effect on the dislocation and the annihilating velocity of moving dislocation was reduced to some extent. In addition, pulse electric current had beneficial effect on the movement and multiplication of dislocation. The coexisting effect of above-mentioned factors would result in the increase of dislocation density. The X-ray diffraction result, as listed in Table 1, shows that the dislocation density is at high level of $3.6 \times 10^{12} \mathrm{~cm}^{-1}$ in the electrostimulated specimen. On the other hand, pulse electric current would be concentrated at the projected part with high curvature of carbide, which resulted in much more remarkable Joule heating effect, and the carbide resolved at the projected edge. Therefore, the interface between carbide and martensite matrix was much improved under the function of pulse electric current, which was beneficial for the improvement of thermal fatigue properties of CHWD steel.

\subsection{Tensile Properties of $\mathrm{HAZ}$}

In order to further study the role of pulse electric current stimulation on the mechanical properties of material, the tensile curves of the CHWD steels with different treatment measures are illustrated in Fig. 4. The profile shows that, after the pulse electric current stimulation, the UTS values of the electrostimulated specimens are improved by $39 \%$ and $55 \%$ for the original and fatigued steels, respectively. The yield strength also increases with a similar trend after pulse electric current. The reasons were regarded the collective actions of the following strengthening mechanisms.

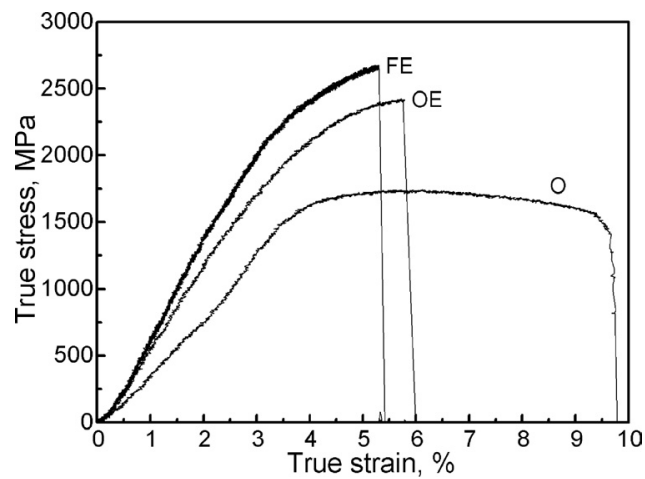

Fig. 4. Tensile curve of CHWD steels untreated and treated by pulse electric current stimulation.

First of all, the strengthening mechanism by refining grains had a remarkable role on the improvement of UTS of electrostimulated specimens. As repeatedly reported in our former papers, ${ }^{22,23)}$ the discharging duration was extremely short and the rapid heating was beneficial for increasing grain nucleation rate. Because temperature of matrix was relatively low, the rapid heat exchange occurred between heating HAZ and cooling matrix. The nucleated grains could not grow for lack of favorable dynamic conditions during the consequent rapid cooling process. As a result, the martensite grains/subgrains in the electrostimulated specimen HAZ were remarkably refined from lath morphology in original state to ultrafine particle. ${ }^{22)}$ The grain refining mechanism was enhanced by pulse electric current stimulation, which resulted in the increase of UTS of electrostimulated specimens. Secondly, when the induced thermal stress, accompanied by electron wind force, could not exceed the yield strength of material, the induced energy would promote the mobility of existing dislocations under pulse electric current stimulation. ${ }^{11)}$ Once the moving dislocation surpassed second-phase carbide particle, dislocation loops were produced in the regions where the dislocation slipping and climbing occurred, which resulted in the increase of dislocation density, namely that Orowan dislocation multiplication phenomenon. The resistance of plastic deformation was enhanced due to the existence of high density dislocation in the electrostimulated specimens and the strength of material would be improved remarkably. Thirdly, a great deal of nanosized carbide particles precipitated and dispersively distributed in the electrostimulated specimens after the pulse electric current stimulation. As a rule, the more dispersive was the distribution of nanosized carbide particles, the more dislocation would multiply in the bulk of material. Therefore, the pulse electric current stimulation was beneficial for the dispersive distribution of newly formed carbide particles and the multiplication of moving dislocations in the HAZ, which ultimately resulted in the increase of tensile strength and yield strength of electrostimulated specimens.

The difference of UTS between the original specimen and the fatigued one after pulse electric current stimulation was regarded as the result of microstructural changes and increasing dislocation density during thermal fatigue cycle. During the heating process for $60 \mathrm{~s}$, the rate of temperature rise on the specimen surface was rather higher than that in the specimen inner. The expanding of specimen surface was 

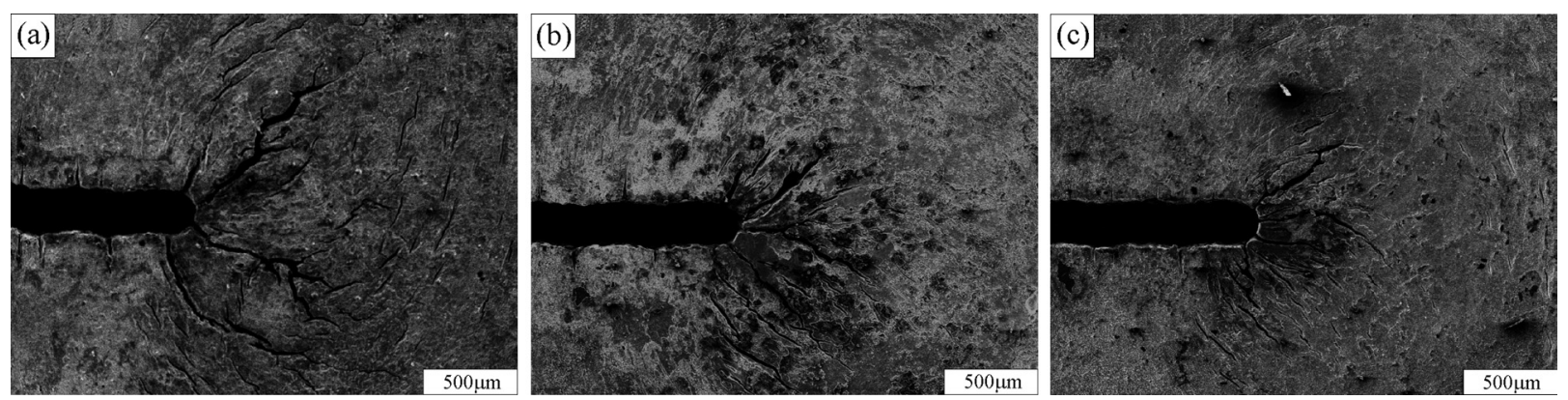

Fig. 5. Fatigue cracks of CHWD steels with different treatments: (a) O, (b) OE, and (c) FE after 2000 thermal cycles.

restrained by the inner of low temperature. When the specimen was cooled in the water, the cooling rate on the specimen surface was relatively higher than that in the specimen inner. The shrinking of specimen surface was restrained by the inner of high temperature. Due to the non-uniform temperature distribution on the specimen, the thermal stress was induced in both the above-mentioned processes. As above-mentioned, the plastic deformation induced by thermal stress was serving as the driving force for the coarsening of carbide particles. Large amount of coarsening carbide particles would be present in the fatigued specimen, as shown in Fig. 3(c). However, the pulse electric current was much more sensitive to carbide particles than martensite matrix. The coexisting action of electron wind force, thermal release and thermal stress would result in the dissolution and redistribution of carbide particles. In addition, pulse electric current enhanced the movement and multiplication of dislocation. The dispersive distribution of plentiful nanosized carbide particles, as illustrated in Fig. 3(c), was beneficial for the stabilization of dislocation substructure. Compared with the specimen electrostimulated in the original state, the fatigued specimen with the application of pulse electric current after 500 thermal cycles was rich in dispersive nanosized carbide particles and stabilized dislocation substructure. Therefore, when the pulse electric current was passing through the original and fatigued specimens, the strengthening effect of the latter was much more remarkable than that of the former.

It was obviously observed from the tensile curves, as illustrated in Fig. 4, that the elongation of electrostimulated specimen was much lower than the unelectrostimulated one, and the elongation reduction was much more remarkable for the fatigued specimen after pulse electric current. The reason was regarded as that, compared with the original specimen, the redistribution of carbide particles increased the resistance of dislocation movement and plastic deformation in the electrostimulated one in the original state. The distribution of nanosized carbide particles was much more dispersive after the pulse electric current stimulation on the fatigued specimen, which would further increase the resistance of dislocation movement and plastic deformation. Therefore, after pulse electric current stimulation, the elongation was reduced in the original and fatigued specimens, especially for the latter.

\subsection{Results of Thermal Fatigue Test}

The fatigue crack morphologies of CHWD steels with different treatments: (a) O, (b) OE, and (c) FE after 2000

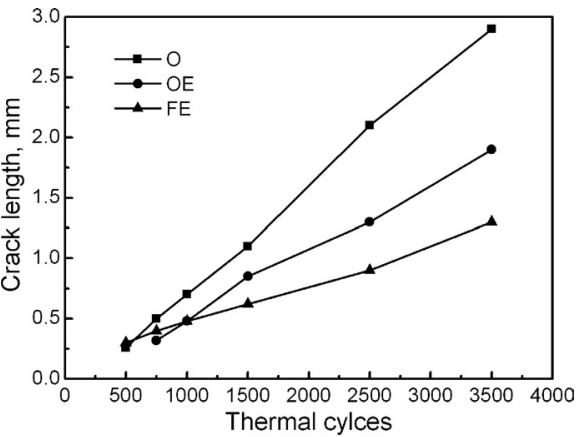

Fig. 6. Main crack length of CHWD steels untreated and treated by pulse electric current at different thermal fatigue cycles.

thermal cycles, are shown in Fig. 5. It was obviously observed that the maximum crack lengths of the electrostimulated specimens were much shorter than that of the unelectrostimulated one. In addition, the maximum crack length of specimen electrostimulated in original state was much shorter than that of specimen electrostimulated in the intermediate stage of thermal fatigue cycle. The pulse electric current stimulation was beneficial for increasing the resistance against thermal fatigue and consequently prolonging the service lifetime of material. The maximum thermal fatigue crack length during thermal cycles was depicted in Fig. 6. It could be seen that the thermal crack had initiated ahead of the notch of original and unelectrostimulated specimen at 500 thermal cycles, while the initiation of the specimen electrostimulated in original state was delayed for 250 thermal cycles and approximately at 750 thermal cycles. Pulse electric current stimulation delayed the initiation of thermal crack. The possible reason was regarded as the improvement of UTS of original specimen induced by pulse electric current stimulation increased the resistance of thermal crack initiation. Similarly, the resistance of thermal crack propagation was also enhanced by increasing the strength of material after pulse electric current stimulation in the original state. Therefore, the propagating rate of thermal fatigue crack was reduced by pulse electric current stimulation, which was testified that the slope of OE curve was much smaller than that of $\mathrm{O}$ curve in Fig. 6. The strengthening effect was much more remarkable and the resistance against the propagating thermal crack was further increased after the application of pulse electric current on the fatigued specimen. The propagating rate of thermal fatigue crack on the fatigued specimen electrostimulated after 500 thermal cycles was much higher than that of the 
one on the original specimen electrostimulated before thermal cycle, as illustrated in Fig. 6 that the slope of OE curve was much higher than that of FE curve. Therefore, the against the initiation and propagation of thermal crack was enhanced after the application of pulse electric current through CHWD steel in the original state, and the enhancement effect was much more remarkable when the fatigued specimen was stimulated by pulse electric current. In order to prolong the service lifetime of CHWD steel to great extent, the application of pulse electric current stimulation was much more alternative in the intermediate stage of thermal cycle.

\section{Conclusions}

(1) Pulse electric current stimulation promoted the redistribution and dispersive distribution of carbide particles in the HAZs of CHWD steels in the original state and in the intermediate stage of thermal cycles, respectively. The movement and multiplication of dislocation substructure were enhanced by the application of pulse electric current on the CHWD steel in the original state, especially for the fatigued steel after pulse electric current stimulation.

(2) The dislocation density increased in the HAZ of original CHWD steel after pulse electric current stimulation, especially in the fatigued steel. The ultra tensile strength values of electrostimulated specimens were much improved by pulse electric current stimulation. The strengthening effect was much more effective in the fatigued specimen after pulse electric current stimulation.

(3) Pulse electric current stimulation increased the resistance of the initiation and propagation of thermal crack, and the enhancement effect was much more effective for the fatigued specimen after pulse electric current stimulation. The application of pulse electric current stimulation in the intermediate stage of thermal cycle was much more alternative for prolonging the service lifetime of the CHWD steel.

\section{Acknowledgements}

This work is supported by the Research Fund for Doctoral Program of Higher Education (No. 20050183053), the
863 Program (No. 2006AA03Z566) granted by the Ministry of Science and Technology of the People's Republic of China and Project 985-Automotive Engineering of Jilin University.

\section{REFERENCES}

1) L. Felberbaum, K. Voisey, M. Gaumann, B. Viguier and A. Mortensen: Mater. Sci. Eng. A, A299 (2001), 152.

2) A. Persson, S. Hogmark and J. Bergstrom: Surf. Coat. Technol., 191 (2005), 216.

3) H. Conard: Mater. Sci. Eng. A, A287 (2000), 205.

4) X. D. Shi, J. S. Wang, Q. G. Xue and D. Q. Zang: Rare Met., 24 (2005), No. 3, 288.

5) M. Nakada, Y. Shiohara and M. C. Flemings: ISIJ Int., 30 (1990), No. 1, 27.

6) M. Gao, G. H. He, F. Yang, J. D. Guo, Z. X. Yuan and B. L. Zhou: Mater. Sci. Eng. A, A337 (2002), 110.

7) H. Conard: Mater. Sci. Eng. A, A287 (2000), 227.

8) Z. H. Xu, G. Y. Tang, S. Q. Tian and J. C. He: Mater. Sci. Eng. A, A424 (2006), 300

9) X. H. Li, Z. S. Gao, W. Li, K. W. Zhang, J. W. Zhang and X. Y. Zhang: Mater. Lett., 59 (2005), 2782.

10) Z. H. Lai, H. Conard, G. Q. Teng and Y. S. Chao: Mater. Sci. Eng. A, A287 (2000), 238

11) W. Zhang, Y. Z. Zhou, M. L. Sui, G. H. He, J. D. Guo and D. X. Li: J. Mater. Sci. Lett., 21 (2002), 1923.

12) Y. Z. Zhou, S. H. Xiao and J. D. Guo: Mater. Lett., 58 (2004), 1948.

13) S. H. Xiao, J. D. Guo, S. D. Wu, G. H. He and S. X. Li: Scr. Mater, 46 (2002), 1.

14) H. Conard: Mater. Sci. Eng. A, A287 (2000), 276.

15) Z. Y. Liu, X. C. Xu and J. Z. Cui: Trans. Nonferrous Met. Soc. China, 13 (2003), No. 4, 743.

16) Z. Y. Liu, Y. Lei and S. D. Li: Trans. Nonferrous Met. Soc. China, 10 (2000), No. 1, 39.

17) O. V. Sosnin, A. V. Gromova, Y. F. Ivanov, S. V. Konovalov, V. E. Gromov and E. V. Kozlov: Int. J. Fatigue, 27 (2005), 1186.

18) O. V. Sosnin, A. V. Gromova, E. Yu. Suchkova, E. V. Kozlov, Yu. F. Ivanov and V. E. Gromov: Int. J. Fatigue, 27 (2005), 1221.

19) O. V. Sosnin, Y. F. Ivanov, V. E. Gromov, E. V. Kozlov, V. V. Tsellermaer and E. Y. Suchkova: Fiz. i Khim. Obrab. Mater., 4 (2003), 63.

20) L. B. Zuev, O. V. Sosnin, S. F. Podboronnikov, V. E. Gromov and S. N. Gorlova: Solids, 45 (2000), No. 3, 24.

21) V. V. Levitin and S. V. Loskutov: Solid State Commun., 131 (2004), 181.

22) H. Q. Lin, Y. G. Zhao, Z. M. Gao and L. G. Han: Mater. Sci. Eng. A, A (2007), doi:10.1016/j.msea. 2007.05.077.

23) Y. G. Zhao, Y. H. Liang, W. Zhou, Q. D. Qin and Q. C. Jiang: ISIJ Int., 45 (2005), No. 3, 410. 